Bull. Korean Math. Soc. 51 (2014), No. 6, pp. 1649-1654

http://dx.doi.org/10.4134/BKMS.2014.51.6.1649

\title{
THE NUMBER OF PANCYCLIC ARCS CONTAINED IN A HAMILTONIAN CYCLE OF A TOURNAMENT
}

\author{
Michel Surmacs
}

\begin{abstract}
A tournament $T$ is an orientation of a complete graph and an $\operatorname{arc}$ in $T$ is called pancyclic if it is contained in a cycle of length $l$ for all $3 \leq l \leq n$, where $n$ is the cardinality of the vertex set of $T$. In 1994, Moon [5] introduced the graph parameter $h(T)$ as the maximum number of pancyclic arcs contained in the same Hamiltonian cycle of $T$ and showed that $h(T) \geq 3$ for all strong tournaments with $n \geq 3$. Havet [4] later conjectured that $h(T) \geq 2 k+1$ for all $k$-strong tournaments and proved the case $k=2$. In 2005, Yeo [7] gave the lower bound $h(T) \geq \frac{k+5}{2}$ for all $k$-strong tournaments $T$. In this note, we will improve his bound to $h(T) \geq \frac{2 k+7}{3}$.
\end{abstract}

\section{Introduction and terminology}

We use Bang-Jensen and Gutin [1] for terminology and notation not defined here. A tournament $T$ is an orientation of a complete graph. We denote by $V(T)$ and $A(T)$ the vertex set and arc set of $T$, respectively. For convenience, let $n$ be the cardinality of $V(T)$. If $x y \in A(T)$, we mostly use the notation $x \rightarrow y$ to denote this arc.

Let $X$ be a subset of $V(T)$. The subdigraph of $T$ induced by $X$ is denoted by $T[X]$. Instead of $T[V(T) \backslash X]$, we write $T-X$ (or $T-x$ if $X$ contains only a single vertex $x$ ). For a vertex $x \in X$, the out-neighborhood (in-neighborhood, respectively) in $T[X]$ is the set $N_{T[X]}^{+}(x)=\{y \mid x y \in A(T[X])\}\left(N_{T[X]}^{-}(x)=\right.$ $\{y \mid y x \in A(T[X])\}$, respectively). Instead of $N_{T}^{+}$and $N_{T}^{-}$we use $N^{+}$and $N^{-}$, respectively. For a subset $Y$ of $X$, we define $N_{T[X]}^{+}(Y)=\bigcup_{x \in Y} N_{T[X]}^{+}(x) \backslash Y$ and $N_{T[X]}^{-}(Y)=\bigcup_{x \in Y} N_{T[X]}^{-}(x) \backslash Y$. We call $d_{T[X]}^{+}(x)=\left|N_{T[X]}^{+}(x)\right|\left(d^{+}(x)\right.$, respectively) the out-degree and $d_{T[X]}^{-}(x)\left(d^{-}(x)\right.$, respectively) the in-degree of a vertex $x \in X$. Also, $\delta^{+}=\delta^{+}(T)=\min \left\{d^{+}(x) \mid x \in V(T)\right\}$ is the minimum out-degree in $T$.

By a path or a cycle, we mean a directed path or directed cycle. An l-cycle in $T$ is a cycle of length $l$. An $n$-cycle is also referred to as a Hamiltonian cycle.

Received August 29, 2013; Revised March 24, 2014

2010 Mathematics Subject Classification. 05C20.

Key words and phrases. tournament, pancyclic arc, Hamiltonian cycle. 
An $\operatorname{arc} e=x y \in A(T)$ is called an out-arc of the vertex $x$. Furthermore, it is called pancyclic in $T$, if it is contained in an $l$-cycle for all $3 \leq l \leq n$.

A strong component $H$ of $T$ is a maximal subdigraph such that for any $x, y \in V(H)$, there is a path from $x$ to $y$ in $H$ and vice versa. If a tournament $T$ has only one strong component, we call it strongly connected or strong. $T$ is called $k$-strong, if $T-X$ is strong for any $X \subseteq V(T)$ with at most $k-1$ vertices.

In 1994, Moon [5] introduced the graph parameter $h(T)$ as the maximum number of pancyclic arcs contained in the same Hamiltonian cycle of a tournament $T$ and proved the next theorem.

Theorem 1.1 ([5]). Let $T$ be a strong tournament on $n \geq 3$ vertices. Then

$$
h(T) \geq 3 \text {. }
$$

The subject was studied for $k$-strong tournaments with $k \geq 2$ by Havet [4], in 2004. With $h_{k}(n)$ defined as

$$
h_{k}(n):=\min \{h(T) \mid T \text { is a } k \text {-strong tournament of order } n\}
$$

(or $h_{k}(n):=\infty$, respectively, if there is no $k$-strong tournament of order $n$ ), he gave the following conjecture.

Conjecture $1.2([4]) . h_{k}(n) \geq 2 k+1$. Given a sufficiently large integer $n$, $h_{k}(n)=3 k$ holds.

Furthermore, he proved his lower bound conjecture for $k=2$.

Theorem $1.3([4])$. Let $T$ be a 2-strong tournament. Then

$$
h(T) \geq 5 \text {. }
$$

The best known lower bound for $k \geq 3$, prior to this note, is due to Yeo [7], who showed the following, in 2005.

Theorem $1.4([7])$. $h_{k}(n) \geq \frac{k+5}{2}$ for $k \geq 1$.

In this note, we will improve his bound to $h_{k}(n) \geq \frac{2 k+7}{3}$ for $k \geq 1$.

But first we will give some results on tournaments we will use to prove our proposition.

\section{Preliminaries}

We begin with a well-known theorem by Camion [2].

Theorem 2.1 ([2]). A tournament is strong if and only if it has a Hamiltonian cycle.

Theorem 2.2 ([6]). If e is an arc of a 3 -strong tournament, then e is contained in a Hamiltonian cycle of $T$.

Lemma $2.3([7])$. Let $T$ be a 2-strong tournament, containing an arc $e=x y$, such that $d^{+}(x) \leq d^{+}(y)$. Then $e$ is pancyclic in $T$. 
Theorem $2.4([7])$. Let $T$ be a 3-strong tournament, containing an arc $e=x y$, such that $d^{+}(x)=\delta^{+}(T)$ and $d^{+}(y)=\min \left\{d^{+}(w) \mid w \in N^{+}(x)\right\}$. Then all out-arcs of $x$ and all out-arcs of $y$ are pancyclic.

Lemma 2.5 ([3]). Let $T$ be a 3-strong tournament, containing an arc $e=x y$, such that $d^{+}(x)=\delta^{+}(T), d^{+}(y)=\min \left\{d^{+}(w) \mid w \in N^{+}(x)\right\}$ and $\mid\{v \in V(T) \mid$ $\left.d^{+}(v)=\delta^{+}(T)\right\} \mid \leq 2$. If
A. $N^{+}(x) \cap N^{+}(y) \neq \emptyset \quad$ or
B. $N^{+}(x) \cap N^{+}\left(N^{+}(y)\right) \neq \emptyset$

then there exists a vertex $z \in N^{+}(x) \backslash\{y\}$, such that all out-arcs of $z$ are pancyclic.

Lemma 2.6. Let $T$ be a k-strong tournament and let $S$ be a subset of $V(T)$ with $s=|S| \leq k-2$. If $e$ is a pancyclic arc in $T-S$, then $e$ is pancyclic in $T$.

Proof. Since $e$ is pancyclic in $T-S, e$ is contained in cycles of length $l$ for all $3 \leq l \leq n-s$. For $n-s+1 \leq l \leq n$, let $S^{\prime}$ be a subset of $S$ with $\left|S^{\prime}\right|=n-l \leq n-(n-s+1)=s-1 \leq \bar{k}-3$. Then $T-S^{\prime}$ is 3 -strong and therefore, by Theorem 2.2, $e$ is contained in a Hamiltonian cycle of $T-S^{\prime}$, which is a cycle of length $l$ in $T$.

Lemma 2.7. Let $T$ be a $k$-strong $(k \geq 5)$ tournament, containing three vertices $x_{0}, x_{1}, x_{2}$, such that all out-arcs of $x_{0}, x_{1}$ and $x_{2}$ are pancyclic. If $T\left[\left\{x_{0}, x_{1}, x_{2}\right\}\right]$ is a 3 -cycle and $h\left(T-\left\{x_{0}, x_{1}, x_{2}\right\}\right) \geq \frac{2(k-3)+7}{3}$, then $h(T) \geq$ $\frac{2 k+7}{3}$.

Proof. Without loss of generality, we may assume that $C_{3}=x_{0} x_{1} x_{2} x_{0}$ is a 3 -cycle in $T$. As a direct consequence of $h\left(T-\left\{x_{0}, x_{1}, x_{2}\right\}\right) \geq \frac{2(k-3)+7}{3}$, there is a Hamiltonian cycle $C_{H}=v_{0} v_{1} \cdots v_{n-4} v_{0}$ of $T-\left\{x_{0}, x_{1}, x_{2}\right\}$ that contains at least $\frac{2(k-3)+7}{3}$ arcs which are pancyclic in $T-\left\{x_{0}, x_{1}, x_{2}\right\}$. By Lemma 2.6, these arcs are also pancyclic in $T$.

Suppose that there are indices $i \in\{0, \ldots, n-4\}$ and $j \in\{0,1,2\}$, such that $v_{i} \rightarrow x_{j}$ and $x_{j+2} \bmod 3 \rightarrow v_{i+1} \bmod n-3$. Without loss of generality, we may assume that $i=j=0$ (up to rotation and relabeling of $C_{H}$ and $C_{3}$ ). Then $v_{0} x_{0} x_{1} x_{2} v_{1} \cdots v_{n-4} v_{0}$ is a Hamiltonian cycle of $T$ that contains at least $\frac{2(k-3)+7}{3}-1+3=\frac{2 k+7}{3}$ pancyclic arcs, since we lose only the arc $v_{0} v_{1}$ and gain 3 pancyclic out-arcs of $x_{0}, x_{1}$ and $x_{2}$ in comparison to $C_{H}$.

Suppose now that there are no such indices and let us denote this property by $(\star)$. If $\left|N^{+}\left(v_{i_{0}}\right) \cap\left\{x_{0}, x_{1}, x_{2}\right\}\right|=3$ for some index $i_{0} \in\{0, \ldots, n-4\}$, we have $v_{i} \rightarrow\left\{x_{0}, x_{1}, x_{2}\right\}$ for all $i \in\{0, \ldots, n-4\}$, by $(\star)$. Hence, $T$ is not strong, a contradiction. By symmetry, the same contradiction is reached if $\left|N^{+}\left(v_{i_{0}}\right) \cap\left\{x_{0}, x_{1}, x_{2}\right\}\right|=0$ (i.e., $\left|N^{-}\left(v_{i_{0}}\right) \cap\left\{x_{0}, x_{1}, x_{2}\right\}\right|=3$ ) for some index $i_{0} \in\{0, \ldots, n-4\}$. It follows that $\left|N^{+}\left(v_{i}\right) \cap\left\{x_{0}, x_{1}, x_{2}\right\}\right| \in\{1,2\}$ for all $i \in\{0, \ldots, n-4\}$. Without loss of generality, we may assume that $N^{+}\left(v_{0}\right) \cap\left\{x_{0}, x_{1}, x_{2}\right\}=\left\{x_{0}, x_{1}\right\}$. Otherwise, we consider the converse tournament (where the direction of all arcs is reversed) and/or relabel the vertices $x_{0}$, 
$x_{1}$ and $x_{2}$, respectively. Because of $(\star)$ and $\left|N^{+}\left(v_{i}\right) \cap\left\{x_{0}, x_{1}, x_{2}\right\}\right| \in\{1,2\}$, we then have $N^{+}\left(v_{i}\right)=\left\{x_{-i} \bmod 3, x_{1-i} \bmod 3\right\}$ for all $i \in\{0, \ldots, n-4\}$.

Suppose that there is an index $i \in\{0, \ldots, n-4\}$, such that $v_{i} v_{i+1} \bmod n-3$ is not pancyclic in $T$. Without loss of generality, we may assume that $i=0$. Then $C=v_{0} x_{0} x_{1} v_{1} \cdots v_{n-4} v_{0}$ is a Hamiltonian cycle of $T-x_{2}$ that contains at least $\frac{2(k-3)+7}{3}+2=\frac{2 k+7}{3}$ pancyclic arcs. We relabel $C=w_{0} \ldots w_{n-2} w_{0}$. Then, since $T$ is strong, there is an index $j \in\{0, \ldots, n-2\}$, such that $w_{j} \rightarrow$ $x_{2} \rightarrow w_{j+1} \bmod n-1$. Therefore, we can insert $x_{2}$ into $C$. Thereby, we lose at most one pancyclic arc (specifically $\left.w_{j} w_{j+1} \bmod n-1\right)$ of $C$ and gain another one (the out-arc of $x_{2}$ ). Thus, we obtain a Hamiltonian cycle of $T$ that still contains at least $\frac{2 k+7}{3}$ pancyclic arcs.

If all arcs of $C_{H}$ are pancyclic, then $C_{H}$ contains $n-3 \geq 2 k+1-3 \geq k+3 \geq$ $\frac{2 k+7}{3}$ pancyclic arcs, since $T$ is $k$-strong $(k \geq 5)$. As seen above, we can insert $x_{0}, x_{1}$ and $x_{2}$ individually into $C_{H}$ without reducing the number of pancyclic arcs contained in the resulting cycle.

\section{Main result}

Theorem 3.1.

$$
h_{k}(n) \geq \frac{2 k+7}{3} \text { for } k \geq 1 .
$$

Proof. We will prove the theorem by induction on $k$. We already know that it is true for $k \in\{1,2,3,4\}$ from Theorem 1.1 and Theorem 1.3. So let $T$ be a $k$-strong tournament with $k \geq 5$ and let $M=\left\{x \in V(T) \mid d^{+}(x)=\delta^{+}(T)\right\}$.

Case 1. $|M| \geq 3$. Let $x_{0}, x_{1}, x_{2} \in M$.

By Lemma 2.3, we have that all out-arcs of $x_{1}, x_{2}$ and $x_{3}$ are pancyclic. Since $T$ is $k$-strong $(k \geq 5), T-\left\{x_{0}, x_{1}, x_{2}\right\}$ is a $(k-3)$-strong tournament. Thus, by the induction hypothesis, there is a Hamiltonian cycle $C_{H}=v_{0} v_{1} \cdots v_{n-4} v_{0}$ in $T-\left\{x_{0}, x_{1}, x_{2}\right\}$ that contains at least $\frac{2(k-3)+7}{3}$ arcs which are pancyclic in $T-\left\{x_{0}, x_{1}, x_{2}\right\}$. By Lemma 2.6, these arcs are also pancyclic in $T$. If $T\left[\left\{x_{0}, x_{1}, x_{2}\right\}\right]$ is a 3 -cycle, then Lemma 2.7 gives us the result.

Thus, without loss of generality, we may assume that $T\left[\left\{x_{0}, x_{1}, x_{2}\right\}\right]$ is not a 3 -cycle and $x_{i} \rightarrow x_{j}$ for all $0 \leq i<j \leq 2$. If there is no index $i \in\{0, \ldots, n-4\}$, such that $x_{2} \rightarrow v_{i+1} \bmod n-3$ and $v_{i} \rightarrow x_{0}$, then $x_{0}$ has at least as many outneighbors in $V(T) \backslash\left\{x_{0}, x_{1}, x_{2}\right\}$ as $x_{2}$. Therefore, we have

$$
\begin{aligned}
d^{+}\left(x_{0}\right) & =d_{T-\left\{x_{1}, x_{2}\right\}}^{+}\left(x_{0}\right)+d_{T\left[\left\{x_{0}, x_{1}, x_{2}\right\}\right]}^{+}\left(x_{0}\right) \\
& =d_{T-\left\{x_{1}, x_{2}\right\}}^{+}\left(x_{0}\right)+2>d_{T-\left\{x_{0}, x_{1}\right\}}^{+}\left(x_{2}\right)+0 \\
& =d_{T-\left\{x_{0}, x_{1}\right\}}^{+}\left(x_{2}\right)+d_{T-\left\{x_{0}, x_{1}, x_{2}\right\}}^{+}\left(x_{2}\right)=d^{+}\left(x_{2}\right),
\end{aligned}
$$

a contradiction to $d^{+}\left(x_{0}\right)=d^{+}\left(x_{2}\right)=\delta^{+}(T)$. Thus, there is such an index $i$ and we obtain a Hamiltonian cycle of $T$ that contains at least $\frac{2 k+7}{3}$ pancyclic arcs as in Lemma 2.7.

Case 2. $|M| \leq 2$. 
If $|M|=2$, let $x_{0}, x_{1} \in M$, such that $x_{0} \rightarrow x_{1}$. If $|M|=1$, let $x_{0} \in M$ and $x_{1} \in N^{+}\left(x_{0}\right)$, such that $d^{+}\left(x_{1}\right)=\min \left\{d^{+}(w) \mid w \in N^{+}\left(x_{0}\right)\right\}$. From Theorem 2.4 we know that all out-arcs of $x_{0}$ and $x_{1}$ are pancyclic.

Suppose that $N^{+}\left(x_{0}\right) \cap N^{+}\left(x_{1}\right) \neq \emptyset$ or $N^{+}\left(x_{0}\right) \cap N^{+}\left(N^{+}\left(x_{1}\right)\right) \neq \emptyset$. Then Lemma 2.5 gives us the existence of an $x_{2} \in N^{+}\left(x_{0}\right) \backslash\left\{x_{1}\right\}$, such that all out-arcs of $x_{2}$ are pancyclic. Without loss of generality, we may assume that $x_{1} \rightarrow x_{2}$. Otherwise, we simply swap $x_{1}$ and $x_{2}$. Thus, we have $x_{i} \rightarrow x_{j}$ for all $0 \leq i<j \leq 2$, a Hamiltonian cycle $C_{H}=v_{0} v_{1} \cdots v_{n-4} v_{0}$ of $T-\left\{x_{0}, x_{1}, x_{2}\right\}$ that contains at least $\frac{2(k-3)+7}{3}$ pancyclic arcs (by the induction hypothesis) and $d^{+}\left(x_{0}\right) \leq d^{+}\left(x_{2}\right)$. Our proposition follows as in Case 1 .

Suppose now that $N^{+}\left(x_{0}\right) \cap N^{+}\left(x_{1}\right)=N^{+}\left(x_{0}\right) \cap N^{+}\left(N^{+}\left(x_{1}\right)\right)=\emptyset$ and let us denote this property by $(\star \star)$. Let $x_{2} \in N^{+}\left(x_{1}\right)$, such that $d^{+}\left(x_{2}\right)=$ $\min \left\{d^{+}(w) \mid w \in N^{+}\left(x_{1}\right)\right\}$ and let $C_{H}=v_{0} v_{1} \cdots v_{n-4} v_{0}$ be a Hamiltonian cycle of $T-\left\{x_{0}, x_{1}, x_{2}\right\}$ that contains at least $\frac{2(k-3)+7}{3}$ pancyclic arcs, whose existence is guaranteed by the induction hypothesis. By $(\star \star)$, we then have $x_{2} \rightarrow x_{0}$. From $d^{+}\left(x_{2}\right)>\delta^{+}(T)=d^{+}\left(x_{0}\right)$, it follows that $x_{2}$ has more outneighbors in $V(T) \backslash\left\{x_{0}, x_{1}, x_{2}\right\}$ than $x_{0}$. Consequently, as seen above, there is an index $i \in\{0, \ldots, n-4\}$, such that $x_{2} \rightarrow v_{i+1} \bmod n-3$ and $v_{i} \rightarrow x_{0}$. Without loss of generality, we may assume that $i=0$ (up to rotation and relabeling of $\left.C_{H}\right)$.

Let us now consider the Hamiltonian cycle $C=v_{0} x_{0} x_{1} x_{2} v_{1} \cdots v_{n-4} v_{0}$ of $T$. If $v_{0} v_{1}$ is not pancyclic in $T-\left\{x_{0}, x_{1}, x_{2}\right\}$, then $C$ contains at least $\frac{2(k-3)+7}{3}+$ $2=\frac{2 k+7}{3}$ pancyclic arcs (those of $C_{H}$ plus the out-arcs of $x_{0}$ and $x_{1}$ ). Suppose that $v_{0} v_{1}$ is pancyclic in $T-\left\{x_{0}, x_{1}, x_{2}\right\}$ (and therefore in $T$ ). We will show that the $\operatorname{arc} x_{2} v_{1}$, then, is also pancyclic in $T$. Consequently, $C$ then contains at least $\frac{2(k-3)+7}{3}-1+3=\frac{2 k+7}{3}$ pancyclic arcs and we have finished.

The pancyclicity of $v_{0} v_{1}$ guarantees the existence of an $l$-cycle

$$
v_{0} v_{1} w_{2} \cdots w_{l-1} v_{0}
$$

in $T-\left\{x_{0}, x_{1}, x_{2}\right\}$ for all $3 \leq l \leq n-3$. Then $x_{2} v_{1} w_{2} \cdots w_{l-1} v_{0} x_{0} x_{1} x_{2}$ is an $(l+3)$-cycle in $T$ that contains $x_{2} v_{1}$ for all $3 \leq l \leq n-3$. Thus, all that remains to be shown is that $x_{2} v_{1}$ is contained in cycles of length 3 to 5 in $T$.

If $x_{1} \rightarrow v_{1}$, then we have $d^{+}\left(v_{1}\right) \geq d^{+}\left(x_{2}\right)=\min \left\{d^{+}(w) \mid w \in N^{+}\left(x_{1}\right)\right\}$ and thus, $x_{2} v_{1}$ is already pancyclic, by Lemma 2.3 . Hence, we may assume that $v_{1} \rightarrow x_{1}$. Then $x_{2} v_{1}$ is contained in the 3-cycle $x_{2} v_{1} x_{1} x_{2}$. Furthermore, $(\star \star)$ gives us $v_{1} \rightarrow x_{0}$ and thus, $x_{2} v_{1}$ is contained in the 4-cycle $x_{2} v_{1} x_{0} x_{1} x_{2}$. Finally, if $v_{2} \rightarrow x_{0}$, then $x_{2} v_{1}$ is contained in the 5-cycle $x_{2} v_{1} v_{2} x_{0} x_{1} x_{2}$. Otherwise, i.e., $x_{0} \rightarrow v_{2}$, we have $v_{2} \rightarrow x_{1}$ by $(\star \star)$, and therefore $x_{2} v_{1}$ is contained in the 5-cycle $x_{2} v_{1} x_{0} v_{2} x_{1} x_{2}$.

Acknowledgement. Financial support by the Excellence Initiative of the German Federal and State Governments is gratefully acknowledged. Furthermore, I am grateful to Yubao Guo for introducing me to this subject and to an anonymous referee for his useful comments. 


\section{References}

[1] J. Bang-Jensen and G. Gutin, Digraphs: Theory, Algorithms and Applications, Springer, London, 2000.

[2] P. Camion, Chemins et circuits hamiltoniens des graphes complets, C. R. Acad. Sci. Paris 249 (1959), 2151-2152.

[3] J. Feng, Hamiltonian Cycles in Certain Graphs and Out-arc Pancyclic Vertices in Tournaments, Ph.D. thesis, 67-68, RWTH Aachen, 2008.

[4] F. Havet, Pancyclic arcs and connectivity in tournaments, J. Graph Theory 47 (2004), no. $2,87-110$.

[5] J. W. Moon, On k-cyclic and pancyclic arcs in strong tournaments, J. Combin. Inform. System Sci. 19 (1994), no. 3-4, 207-214.

[6] C. Thomassen, Hamiltonian-connected tournaments, J. Combin. Theory Ser. B 28 (1980), no. $2,142-163$

[7] A. Yeo, The number of pancyclic arcs in a k-strong tournament, J. Graph Theory 50 (2005), no. 3, 212-219.

Lehrstuhl C Für MATHEMATiK

RWTH AACHEN UNIVERSITY

52062 Aachen, Germany

E-mail address: michel.surmacs@rwth-aachen.de 